\title{
Philonsorbonne
}

4 | 2010

Année 2009-2010

\section{La politique ou la raison désirable chez Spinoza}

André MARTINS

\section{OpenEdition}

Journals

Édition électronique

URL : https://journals.openedition.org/philonsorbonne/287

DOI : $10.4000 /$ philonsorbonne. 287

ISSN : 2270-7336

\section{Éditeur}

Publications de la Sorbonne

\section{Édition imprimée}

Date de publication : 15 mai 2010

Pagination : 109-121

ISBN : $978-2-85944-647-5$

ISSN : 1255-183X

\section{Référence électronique}

André MARTINS, «La politique ou la raison désirable chez Spinoza », Philonsorbonne [En ligne], 4 | 2010, mis en ligne le 02 février 2013, consulté le 11 juin 2021. URL : http://journals.openedition.org/ philonsorbonne/287 ; DOI : https://doi.org/10.4000/philonsorbonne.287

(C) Tous droits réservés 


\title{
La politique ou la raison désirable chez Spinoza
}

\author{
André Martins \\ (Professeur associé à l’Université Fédérale de Rio de Janeiro)
}

Lorsqu'un lecteur de l'Éthique de Spinoza lit son Traité théologicopolitique, en particulier la partie consacrée à la politique (chap.16 à 20), il n'est pas rare qu'il éprouve un sentiment d'étrangeté. Il n'y voit sans doute pas immédiatement les principales thèses spinoziennes présentes dans l'Éthique. Au contraire, certaines affirmations de Spinoza évoquent plutôt Hobbes et son Léviathan, ou tout simplement peuvent sembler, peut-être à juste titre, traiter de morale plutôt que d'éthique. C'est d'ailleurs cette étrangeté qui fait problème, comme P.-F. Moreau le fait remarquer : «Dieu, l'esprit, le désir, la morale : il semble bien que Spinoza tranche sur toutes ces questions avec l'attitude dominante de son temps. Il y a cependant un point où on pourrait penser l'en rapprocher : sa théorie politique, dont le terme central est celui de droit naturel, qui est précisément une des notions de l'espace volontariste. Aurait-il ici cédé à l'analyse qui se retrouve, avec des variantes, chez Hobbes, Locke, Rousseau ? » ${ }^{1}$. Dans la seconde partie du Traité théologico-politique, Spinoza analyse à sa manière le contrat ou le pacte social, tel qu'il était conçu par les philosophes anglais de son époque comme Locke et Hobbes. L'idée d'un contrat social signifiait que «les individus abandonnent leur droit naturel à la société qu'ils constituent, afin qu'elle ait le plus de puissance possible pour les protéger contre les méfaits de la Nature et des autres hommes $»^{2}$. P.-F. Moreau relève deux différences entre l'analyse de Spinoza au sujet du contrat social et celle de ses contemporains : 1/ Spinoza s'occupe du «fonctionnement réel qui le sous-

1. P.-F. Moreau, Spinoza, Paris, Seuil, 1975, p. 113.

2. Nous reprenons ici la description qu'en donne P.-F. Moreau, Spinoza et le spinozisme, Paris, PUF, 2003, p. 65. 
tend $»^{3} ; 2$ Il abandonne l'aspect « volontariste» de ces théories, dans le sens où il ne pense pas, contrairement à ses pairs, que les passions sont abandonnées une fois le contrat établi, c'est-à-dire une fois que l'homme aurait quitté l'état de nature et serait passé à l'état civil. Au contraire, l'homme garde toujours son droit naturel et les passions en font partie, si bien qu'elles ne disparaîtront pas après le pacte ${ }^{4}$. Si l'on développe ces deux points afin d'éclairer la différence entre la théorie volontariste du contrat social et l'analyse spinozienne du pacte social, il en découle un troisième point distinctif de la théorie politique de Spinoza que nous essaierons de mettre en évidence, à savoir : 3/ la proposition d'un État qui ne se contente pas de contrer les passions, mais s'intéresse aussi à favoriser les actions.

Nous pensons que les affirmations de Spinoza dans le Traité théologicopolitique, en particulier, et sur la politique en général doivent être comprises à l'intérieur de sa philosophie, en prenant en compte son Éthique et ses concepts. La question se pose de savoir s'il y a cohérence ou coupure entre les domaines éthique et politique. Autrement dit, l'État est-il obligé, pour assurer sa continuité et la paix du peuple, d'agir au détriment de l'éthique afin d'atteindre ses buts, aussi rationnels ou raisonnables soient-ils ?

Comprendre le «fonctionnement réel » qui sous-tend le pacte social implique la critique des théories volontaristes du contrat social. Si l'on veut le comprendre, comme le confirme le célèbre $\$ 1$ du Traité politique à la suite du Traité théologico-politique, on doit prendre en compte les affects humains tels qu'ils existent dans la nature humaine, et non pas comme des défauts à déplorer. Spinoza veut attirer l'attention sur le fait qu'il n'y a pas d'être humain sans affects, ni même sans passions. On ne devrait donc pas s'attendre à ce que les passions disparaissent après l'institution d'un contrat social. Ici, pourtant, quelques rappels à la théorie spinozienne nous semblent nécessaires. Lorsque Spinoza critique durement «les philosophes » qui veulent par une théorie politique morale ou par l'idée d'un contrat social changer la nature de l'homme en lui ôtant ses passions, il faut rappeler qu'il n'emploie pas le même terme qu'eux: dans sa critique, il montre que l'homme ne peut pas se délivrer de ce que les autres philosophes, de son époque, qui sont au fond des moralistes, appellent "passions». Mais Spinoza lui-même a créé un concept différent qui annule la distinction qui existait chez les autres philosophes entre le corps et l'esprit. C'est le concept

3. P.-F. Moreau, Spinoza et le spinozisme, op. cit., p. 60.

4. P.-F. Moreau, Spinoza et le spinozisme, op. cit., p. 65-66.

5. «Les affects, dont les humains sont agités, seraient-ils autant de défauts auxquels nous succomberions par notre faute? Telle est l'opinion des philosophes qui prennent le parti soit d'en rire, soit de se lamenter, d'éclater en reproches - voire en malédictions. Ils se figurent, sans doute, accomplir une œuvre sublime et atteindre à la plus haute sagesse en faisant l'éloge renouvelé d'une nature humaine fictive, pour accuser d'autant plus impitoyablement celle qui existe en fait. Car ils ne conçoivent point les hommes tels qu'ils sont, mais tels que leur philosophie les voudrait être. Aussi, au lieu d'une éthique, ont-ils le plus souvent écrit une satire ; quant à leur doctrine politique, elle est toujours inapplicable, elle évoque une sorte de chimère » (Spinoza, Traité Politique, § 1, trad. M. Francès, Paris, Gallimard, 1978). 
d'affect $^{6}$ qui se divise en affects actifs et affects passifs. Or, au moment où Spinoza établit cette distinction, il sépare ce qui jusqu'alors demeurait mêlé pour les autres philosophes, qui prétendaient placer d'un côté la raison, mais une raison séparée du corps et par conséquent du monde sensible, et de l'autre les passions - pensées «obscures et confuses » qui se laisseraient influencer par le corps ${ }^{7}$. Autrement dit, les philosophes imaginaient une raison abstraite, mais inexistante, qui aurait la capacité de se détacher du sensible pour agir sur celui-ci, alors que Spinoza propose de comprendre que dans la réalité nous aurons toujours des affects, puisque nous n'existons que dans le monde (ou au sens plus large, dans la substance) et par là même sommes toujours affectés ${ }^{8}$, bien que les affects qui en découlent peuvent être passifs ou actifs ${ }^{9}$.

Lorsque Spinoza écrit que l'homme ne peut se passer des passions, faisant ainsi la critique des philosophes moralistes, y compris ceux du contrat social, son affirmation a deux sens : 1/ Tout d'abord, les hommes ne peuvent pas exister sans affects; les hommes existent en chair et en os dans le monde sensible, d'où la première confusion des philosophes qui ne comprennent guère la nature humaine et la rêvent telle qu'elle n'existe pas, délivrée non seulement des passions mais aussi du sensible et du corps. 2/ C'est seulement suite à cette première considération que nous pouvons dire que les hommes ne peuvent pas non plus s'abstenir de façon absolue des passions, au sens spinozien, c'est-à-dire des affects passifs. Car non seulement l'empire de la raison n'est pas absolu face aux passions, mais surtout Spinoza ne partage pas le monde en deux, monde sensible et monde intelligible, res extensa et res cogitans. Il est important de comprendre que la chimère dont parle Spinoza se rapporte en premier lieu à la pensée que l'homme pourrait avoir une raison séparée des affects. Ainsi, le premier point à retenir est que la raison peut contribuer à ce que nos affects soient actifs, dans le cas où notre esprit a des idées adéquates, mais elle ne peut point faire que nous n'ayons pas d'affects du tout.

L'homme ne peut pas soumettre les passions à sa volonté, non pas à cause d'un échec de son esprit ou de sa raison, mais à cause de la nature même et de l'esprit et de la raison, qui sont ici mis en question dans leur acception traditionnelle. En d'autres termes, quand la raison vainc les passions, Spinoza nous rappelle que ce n'est pas qu'elle a vaincu les affects, mais qu'elle a pu favoriser des affects actifs ou transformer des affects passifs en actifs, par un effet de la connaissance, lui-même affectif. L'homme ne peut donc pas se passer des affects. Ce n'est pas la même chose que de dire que l'homme ne peut pas - pour des moments aussi rares ou fréquents soient-ils, pour une courte ou une longue durée - se passer des passions : il peut se passer des passions du moment qu'il a des affects actifs.

6. Spinoza, Éthique, III, déf. 3.

7. Cf. Descartes, Méditation sixième, 63, 66, 71 ; Discours de la méthode, partie IV.

8. Spinoza, Éthique, III, postulat 1.

9. Ibid., prop 1. 
Alors qu'il ne peut point se passer des affects - puisque étant autant esprit que corps, il a toujours des affections, il est toujours affecté. Le pouvoir de la raison ne consistera donc jamais à séparer l'homme des affects, mais, dans les moments de réussite, seulement à ne pas avoir des affects passifs. Enfin, si l'on se limitait à dire que l'homme ne peut pas abandonner ses passions tel que l'ont fait certains philosophes postérieurs à Spinoza, comme Kant et Freud, par exemple - on pourrait croire que les passions appartiennent à une nature plus profonde de l'homme qui s'opposerait à la raison, comme si l'homme était séparé en corps, foncièrement naturel, et esprit, celui-ci devant combattre contre celui-là, en vain d'ailleurs puisque l'esprit ne réussit évidemment jamais son dessein de se délivrer du corps.

Récapitulons, donc. Primo, l'homme ne peut pas ne pas avoir d'affects. Secundo, il ne peut cesser d'avoir des passions, c'est-à-dire des affects passifs ; et cela non pas parce que sa nature serait d'être passionnelle, le rôle de la raison étant de les dominer, mais parce qu'il aura toujours des affects passifs, quand bien même il aurait des affects actifs, affects actifs qui sont donc aussi naturels que les autres - de la même manière que la raison lui est naturelle. Les hommes étant des modifications de la substance unique, leur nature est à la fois active et passive. Active en tant qu'ils sont modes de la nature naturante si bien qu'ils l'expriment ou peuvent l'exprimer ${ }^{10}$, en étant ainsi cause adéquate de ce qui se produit en eux ou hors d'eux ${ }^{11}$; passive puisqu'en étant un mode, donc un individu de la nature naturée, l'homme n'existe qu'en relation avec les autres individus et subit ainsi des affections, étant donné qu'il ne peut être conçu sans les autres parties de la nature ${ }^{12}$. Cette double nature, si l'on peut dire, ne se confond point pour autant avec ce qu'on pourrait appeler la «double nature » traditionnelle qui partage l'homme en raison et passion, esprit et corps, intelligible et sensible. L'homme, tel que le définit Spinoza, sera toujours et nécessairement affectif, mais il peut l'être de façon active, lorsqu'il exprime la nature naturante qui le constitue, aussi bien qu'il peut l'être de façon passive, lorsqu'il se laisse mener par les causes extérieures. Ce n'est pas son esprit qui est actif et son corps passif, mais c'est tout autant son corps et son esprit qui peuvent être passifs ou actifs.

Si l'on suit ce raisonnement selon lequel la liberté d'agir et de penser qu'elle résulte de la raison ou du troisième genre de connaissance - ne peut pas être en dehors de la nature humaine, et encore moins en dehors de la Nature, puisque la nature naturée est la nature naturante et peut donc l'exprimer, alors on pourra mieux comprendre l'idée spinozienne du droit naturel. Celui-ci, d'après le début du chapitre 16 du Traité théologicopolitique, correspond à la puissance naturelle, entendue à la fois comme effective et intrinsèque à chaque individu, qu'il soit un être humain ou un État. Autrement dit, si on raisonne d'abord en pensant à l'homme, puis si on

10. Ibid., IV, prop 4, dém.

11. Ibid., III, déf 2.

12. Ibid., IV, prop 2, dém ; prop 3. 
transpose le raisonnement à l'État, tel que le suggère Spinoza, on risque moins de faire de contresens, c'est-à-dire de tenir ses propos politiques pour une morale et non pour une éthique. Spinoza écrit en effet : "Par Droit et Institution de la Nature, je n'entends autre chose que les règles de la nature de chaque individu, règles suivant lesquelles nous concevons chaque être comme déterminé à exister et à se comporter d'une certaine manière $»^{13}$. Le droit naturel est ainsi défini par Spinoza non pas comme quelque chose qui nous fait sortir de la Nature mais au contraire, comme son expression : le droit naturel suit la singularité de chaque individu. «Par exemple les poissons sont déterminés par la nature à nager », poursuit Spinoza. On pourrait dire: le poisson a la puissance de nager, la capacité, l'habileté effective. Il peut le faire, qu'il le veuille ou pas, c'est un fait, c'est effectif : «c'est-à-dire le Droit de la Nature s'étend aussi loin que s'étend sa puissance », rappelant que « la puissance de la Nature est la puissance même de Dieu». Il s'ensuit que «le droit de chacun s'étend jusqu'où s'étend la puissance déterminée qui lui appartient $»^{14}$. On voit donc que finalement le droit naturel signifie chez Spinoza que nul ne peut, par volonté ou par commandement, aller contre la nature, ou contre sa nature (qui exprime la Nature, Dieu ou la substance) $)^{15}$.

Nous vivons tous, vertueux ou non, raisonnables ou non, « soumis aux seules lois de l'appétit ${ }^{16}$, écrit Spinoza dans le Traité théologico-politique. C'est ce que confirme l'Éthique: ne pas subir les lois de l'appétit est impossible dans le sens où même en suivant la raison ou le troisième genre de connaissance nous suivrons toujours nos appétits et nos désirs ${ }^{17}$. Encore une fois Spinoza se démarque ici des volontaristes, vu que la volonté ou la raison ne peuvent pas s'opposer aux appétits. Par contre, la raison et la connaissance intuitive peuvent faire comprendre à l'individu que ce que ses appétits cherchaient au départ n'était pas ce qui l'épanouirait le mieux. Les appétits, sans le concours de la raison ou de la connaissance intuitive, tendent à la servitude, non pas par une nature servile qui serait celle de l'homme, mais parce que les appétits suivent le plus souvent le hasard des rencontres, déterminés par les causes externes. Autrement dit, les appétits nous guideront toujours, avec ou sans raison. Ce n'est pas que la raison saurait ce qui est le mieux pour l'individu, au détriment des appétits, mais

13. Spinoza, Traité théologico-politique, chap. 16, p. 261 (trad. Charles Appuhn, Paris, GFFlammarion, 1965).

14. Ibid., p. 262.

15. Remarquons que cette nature ne se confond pas avec une idée préalable d'une nature qui serait ainsi idéalisée, fruit de l'imagination érigé en universel, servant de modèle extérieur auquel les particuliers devaient s'adapter.

16. Ibid., p. 262.

17. Spinoza, Éthique, V, prop. 4, scol : «C'est par un seul et même appétit que l'homme est dit tant agir que pâtir. [...] Tous les appétits ou Désirs ne sont pas des passions qu'en tant qu'ils naissent d'idées inadéquates; et ces mêmes Désirs viennent s'adjoindre à la vertu quand ce sont d'idées adéquates qui les excitent ou les engendrent. » (trad. B. Pautrat, Paris, Seuil, 1999). 
que la raison doit aider l'individu à raisonner et à sentir ce qui est le mieux pour lui à chaque instant particulier, dans sa singularité, par et dans l'expérience, par sa science intuitive, selon son ingenium ou complexion. Il est vain que l'individu, unité corps-esprit, cherche à imposer raison à appétit. Il faut qu'ils s'accordent. Sans la raison, les appétits nous trompent - nous désirons des choses qui pourtant n'augmentent pas notre puissance d'agir. En revanche, une supposée « raison » qui ignore la réalité de nos appétits, nous trompe elle aussi - par le même motif. Le volontaristes, par contre, veulent croire que la raison peut imposer sa « vérité » aux appétits, comme l'intelligible imposerait sa vérité au sensible ou au corps, même s'ils peuvent aussi admettre que cela ne se réalise en fait jamais. En bref, dans l'unité corps-esprit spinozienne, la raison n'est pas séparée du corps, si bien qu'elle ne niera pas les appétits, mais peut mener l'homme à mieux comprendre, par des idées adéquates, ce qui lui fait du bien et augmente sa puissance sans être trop troublé par des causes extérieures. Son appétit et son conatus seront alors mieux satisfaits qu'auparavant. Analyser la réalité des passions nous permet de comprendre le besoin d'un État, afin non pas de les empêcher puisqu'elles existeront toujours, mais d'empêcher leur déchirement.

Pourtant, on pourrait encore s'étonner des affirmations de Spinoza concernant les passions de la foule et de ses rapports invivables, la soumission de l'individu «à la violence de ses passions » par lesquelles il juge arbitrairement ce qui lui est utile : «il lui est loisible de l'appéter en vertu d'un Droit de Nature souverain et de s'en saisir par quelque voie que ce soit, par la force, par la ruse, par les prières, enfin par le moyen qui lui paraîtra le plus facile ; conséquemment aussi de tenir pour ennemi celui qui veut l'empêcher de se satisfaire ${ }^{18}$. De plus, le remède trouvé par l'État pour contrecarrer autant de déchaînements serait de faire valoir encore plus de passions. Certes, seule une petite partie des hommes atteint l'état de vertu, mais nous vivons tous, aussi sages que nous puissions être, selon nos appétits et désirs ${ }^{19}$, et personne ne s'affecte suivant des idées adéquates tout le temps, devant tous les évènements qui puissent lui advenir - la raison nous permet aussi de comprendre que nous ne sommes pas rationnels à tout moment ni en toutes circonstances. Certes, on sait bien que dans un peuple il y aura toujours une grande partie de celui-ci qui se trouvera dans l'ignorance, et que la morale sert à faire garder le droit chemin à ceux qui n'ont pas ou pas encore suffisamment développé leur raison.

L'État, analyse Moreau, «peut s'opposer à leurs passions par la force, mais un tel expédient ne peut se prolonger. Il lui faut donc trouver un rempart plus fort : un autre jeu passionnel ou la satisfaction des besoins et des intérêts (encore faudra-t-il convaincre les citoyens que les mesures prises en ce sens vont y parvenir, ce qui renvoie de nouveau aux passions et aux symboles). Parmi les passions que l'État peut utiliser à son profit, on compte évidemment la passion religieuse; mais il s'agit d'une arme à double

18. Spinoza, Traité théologico-politique, chap.16, p. 263.

19. Spinoza, Éthique, III, prop. 9, dém., scol. 
tranchant : on peut exciter les peuples à haïr et massacrer les rois qu'on leur avait enseigné à adorer $»^{20}$. L'Etat, ajoute Cristofolini, peut utiliser des « artifices institutionnels dont le but est d'obtenir que les citoyens soient conduits même malgré eux à agir selon les intérêts de la société tout en restant mus par les ressorts de leurs propres intérêts ${ }^{21}$. En bref, ce que Spinoza dénonce pour le gouvernement monarchique vaut en fait pour toute sorte de gouvernement, même au sein d'une démocratie instituée : «le plus grand secret du gouvernement monarchique et son intérêt principal consistent à tromper les hommes et à masquer du nom spécieux de religion la crainte qui doit les retenir, afin qu'ils combattent pour leur servitude comme si c'était pour leur salut ${ }^{22}$. Mais il se trouve que les passions fomentées par l'État dans le but de maintenir l'unité du peuple auront toujours une limite d'efficacité...

C'est là qu'il faut prendre garde, au moment de lire Spinoza, à la différence entre le constat (la description ou le diagnostic) et la proposition, ou encore, entre la morale constatée et l'éthique proposée. Spinoza constate pourquoi la démagogie, la tyrannie, le patriotisme, la superstition, enfin le maniement des causes extérieures fonctionnent, mais aussi jusqu'à quel point. Le propos de Spinoza, pourtant, au lieu d'être un plaidoyer pour la morale, ou une simple description des moyens passionnels dont l'État peut se servir pour contrer et manier le peuple, consiste à montrer que lorsqu'un peuple a le droit de s'exprimer, il exprime davantage et de façon plus pleine son droit naturel - avec ou sans raison -, et cela à l'intérieur du pacte social (tacite, spontané, ou explicite), si bien que l'État sera préservé d'une révolte provenant d'un excès de frustration de la part de ses membres. Spinoza ne propose pas des moyens passionnels de contrôle de la multitude, il constate leur efficacité, mais aussi leurs limites, pour comprendre les causes affectives autant de son efficacité que de son échec, afin de proposer, au-delà du constat et à partir de l'analyse des causes, des manières de gouvernement et d'institution plus efficaces puisque plus rationnelles.

Spinoza analyse les causes ou la genèse des passions (des affects passifs), au lieu de proposer de les combattre sans comprendre leur fonctionnement, comme si les passions étaient quelque chose qui ne devrait plus exister chez les personnes ou les États rationnels par suite d'un simple acte de la volonté. Mais, on l'a vu, Spinoza ne se contente pas de décrire, précisément parce que cette analyse lui montre la plus grande réalité des affects actifs et la possibilité que l'État contribue à les favoriser. C'est pourquoi il expose ce qu'il entend par démocratie. Par l'explication des passions dans la politique telles qu'elles sont explicitées dans l'Éthique, ce qui vaut pour l'individu humain vaudra aussi, par analogie, pour cet individu qu'est un État ou un peuple. Aussi faut-il que l'État se serve de la raison,

20. P.-F. Moreau, Spinoza et le spinozisme, op. cit., p. 66.

21. P. Cristofolini, Spinoza, chemins dans l'Éthique, Paris, PUF, 1996, p. 63.

22. Spinoza, Traité théologico-politique. Ajoutons que ce n'est pas seulement la religion qui est utilisée à cette fin par les gouvernements, mais les passions en général. 
permettant ainsi que les individus s'expriment dans leur droit naturel et puissent augmenter leur puissance d'agir.

Il serait naïf de croire que la liberté, dans le sens d'une autonomie d'action et de pensée accordée à la puissance de la Nature Naturante, puisse être garantie par la contrainte sur le peuple, ou en ôtant au peuple sa puissance. Cela n'est plus naïf, mais devient une analyse réaliste, si ce n'est plus que le constat d'un fait: que les foules sont passionnelles, que les gouvernants sont obligés de les retenir pour garder le pouvoir et qu'ils peuvent y parvenir par la contrainte, y compris en trompant le peuple. Mais que ce soit pour l'État ou pour les individus, le pouvoir exercé par contrainte sur l'autre vient d'un affect passif, d'une menace imaginaire dont on veut préalablement empêcher la réalisation. Même s'il est compréhensible qu'un État exerce un pouvoir de contrainte sur ses membres comme un droit naturel, cet exercice ne sera pas le fruit d'affects actifs. Que l'État puisse utiliser la force, la crainte ou la croyance pour tromper ses membres, les menant à suivre la morale s'ils n'ont prétendument pas ou pas encore de raison ou d'éthique, ne signifie pas qu'il utilise ainsi la meilleure façon de procéder, aussi bien pour ses membres que pour sa propre puissance.

Spinoza le dit dans l'Éthique ${ }^{23}:$ «Il y a hors de nous bien des choses qui nous sont utiles, et auxquelles, pour cette raison, il faut aspirer. Et parmi elles, on ne peut en imaginer de meilleures que celles qui conviennent entièrement avec notre nature ». Un État qui exige que ses membres abandonnent leurs propres intérêts au nom de l'unité de la société leur demande alors quelque chose qui les aliènent de leur propre puissance. « Si en effet deux individus, par ex., ayant exactement la même nature, se joignent l'un à l'autre, ils composent un individu deux fois plus puissant que chacun pris séparément. À l'homme donc, rien de plus utile que l'homme ». Or, un Etat ne correspondra à un surplus de puissance pour ses membres que si - ou tant que - la puissance de ceux-ci ne diminue pas sous l'effet de l'action qu'il exerce. C'est ce que conclut Spinoza au chapitre 20 du Traité théologico-politique, après avoir analysé auparavant les fondements de l'État : «il ne peut se faire que l'âme d'un homme appartienne entièrement à un autre ; personne en effet ne peut transférer à un autre, ni être contraint d'abandonner son droit naturel ou sa faculté de faire avec sa raison un libre usage et de juger de toutes choses $»^{24}$. Ainsi, il résulte que la fin dernière d'un État «n'est pas la domination; ce n'est pas pour tenir l'homme par la crainte et faire qu'il appartienne à un autre que l'État est institué ; au contraire, c'est pour libérer l'individu de la crainte, pour qu'il [...] conserve, autant que se pourra, sans dommage pour autrui, son droit naturel d'exister et d'agir ${ }^{25}$. »

23. Spinoza, Éthique, IV, prop. 18, scol.

24. Spinoza, Traité théologico-politique, chap.20, p. 327.

25. Ibid., p. 329. 
Il est vrai que tout État n'existe que par un certain degré inévitable de contrainte, ou de contenance, sur les appétits et les passions de tout un chacun, au sens où il doit trouver des lois ou des règles générales pour tous, et non les faire varier selon les perspectives individuelles. Il est néanmoins aussi vrai que cette contrainte de départ s'allège si, comme l'a montré Spinoza $^{26}$, la participation du peuple est plus grande dans les décisions, et si les lois et les règles suivent une régulation et une modulation pour s'adapter au mieux aux cas particuliers, à la jurisprudence et au raisonnement au sujet des singularités qui se présentent, tout en gardant les principes de base.

L'expression individuelle doit, selon Spinoza, être laissée libre de contrainte lorsqu'elle se fait plutôt par des idées que par des actes, mais cette expression ne dépend pas de la raison. Ceci implique que ce ne sera ni l'État, ni sa volonté, ni celle des philosophes, qui diront ce qu'est la vérité de l'autre, ce qui est raisonné et ce qui ne l'est pas, car cela constituerait une morale universelle imposée contre l'autonomie des individus. Si nous comprenons que non seulement les appétits sont extrêmement divers, mais aussi que les jugements sur ce qui est raisonnable dépendent inévitablement de la perspective de celui qui juge, nous comprenons mieux en quoi une vraie démocratie serait la meilleure façon d'avoir une collectivité qui n'empêche pas les puissances ou les droits naturels individuels, mais au contraire les favorise.

Ainsi, le meilleur État sera celui qui favorisera les puissances individuelles, et non point celui qui, au nom de la paix, de la puissance et de l'union de la collectivité, sacrifie la paix et la puissance des individus qui le composent. Un État fort ne peut pas se constituer d'un ensemble d'individus faibles - tristes ou déprimés, passionnels mais conduits à suivre l'État comme une cause extérieure. Au contraire, il faut penser que le règlement inévitable de l'État doit exister autant que possible d'une façon telle qu'elle devienne un support de renforcement des puissances singulières, en fonctionnant comme repère pour chacun, en soutenant les initiatives individuelles, en favorisant leur épanouissement.

En somme, l'État ne doit pas être le but de la collectivité, surtout s'il s'exerce au détriment des personnes, mais un moyen de favoriser le développement individuel de tous ses membres et par là même le développement des idées adéquates de ceux-ci, tout en respectant leur ingenium. Un homme rationnel n'aurait sans doute plus besoin d'un État, s'il vivait tout seul. Mais du fait que chacun juge selon sa complexion de la

26. «Il faut nécessairement accorder aux hommes la liberté du jugement et les gouverner de telle sorte que, professant ouvertement des opinions diverses et opposées, ils vivent cependant dans la concorde. Et nous ne pouvons douter que cette règle de gouvernement ne soit la meilleure, puisqu'elle s'accorde le mieux avec la nature humaine. Dans un État démocratique (c'est celui qui rejoint le mieux l'état de nature) [...], tous conviennent d'agir par un commun décret, mais non de juger et de raisonner en commun ; c'est-à-dire, comme les hommes ne peuvent penser exactement de même, ils sont convenus de donner force de décret à l'avis qui rallierait le plus grand nombre de suffrages, se réservant l'autorité d'abroger les décisions prises sitôt qu'une décision meilleure leur paraîtrait pouvoir être prise » (Ibid., p. 334). 
manière d'utiliser sa pensée, et que la raison est, en tant que connaissance, toujours dynamique, et partant toujours remise en cause en fonction des rencontres, des changements de situation et des conditions où l'on vit, puisqu'on est partie de la nature naturée, un État sera toujours nécessaire pour moduler les différences entre les conceptions individuelles de ce qui est correct, y compris lorsque ces conceptions sont rationnelles - mais aussi pour permettre de mieux vivre ces différences et de mieux les exprimer. La raison - comprenant ce qui est commun, à commencer par le fait que la quête de l'utile est commune à tous et que chacun la réalise à sa manière ne peut empêcher le désaccord, mais elle peut aider à trouver un accord tout en respectant les différences.

Tant qu'ils sont mus par les passions (par les affects passifs), les individus penseront certainement que tout ce que fait l'État les défavorise, si ses actions ne leur apportent pas de profit immédiat, et suivent les intérêts d'autrui, présentés comme s'il s'agissait des intérêts de tous. Or, ils n'ont pas tort : l'État aussi est la plupart du temps mû par les intérêts passionnels et personnels de ses gouvernants, qu'ils soient monarques, présidents, ministres, sénateurs, députés, etc. Autrement dit, le plus souvent le peuple pensera du mal de ses gouvernants, tandis que, le plus souvent, à leur tour, ceux-ci essayeront de tromper le peuple à leur profit, en lui faisant croire qu'ils ont raison et prennent les meilleures mesures, qu'il les comprenne ou non. Dans ce cas, la persuasion devient une règle non plus pour convaincre le peuple irrationnel du bienfait des actions rationnelles des gouvernants, mais pour convaincre le peuple, conduit ou non par la raison, du bienfait des actions passionnelles des gouvernants. De la même façon qu'un individu « juge de ce qui est bon, de ce qui est mauvais, et veille à son intérêt selon son propre tempérament (suo ingenio) ${ }^{27}$, l'État lui aussi juge ce qui est rationnel d'après le tempérament de ses gouverneurs, qui n'est pas exempt de leurs points de vue, passions, appétits et désirs.

Le but d'un État démocratique sera donc bien plus que de seulement préserver sa propre sécurité - autrement dit, de conserver son être à tout prix - mais d'exprimer la puissance de ses membres tout en assurant les conditions (y compris la sécurité en général mais pas seulement) qui favorisent leur expression, leur épanouissement, leur action. S'il est vrai donc que l'État a le droit de faire des lois et d'attendre que les personnes les suivent, cela ne veut pas dire que le seul mécanisme à employer pour les en persuader soit le renforcement des passions ou la contrainte. Il ne faut pas croire que d'un côté, il y a un État raisonnable ou des gouvernants sages et raisonnables, et de l'autre un peuple passionnel et ignorant qui un jour peutêtre pourrait devenir mûr, majeur, adulte et raisonnable, introjectant les règles posées par l'État. Car la raison n'est pas nécessairement du côté de l'Etat. Et la raison est un genre de connaissance qui ne se traduit jamais en des commandements impératifs préalables à l'expérience. Si les personnes ne se laissent pas mener par les raisons soi-disant universelles des

27. Spinoza, Éthique, IV, prop. 37, scol. 2. 
gouvernants, c'est malgré tout un bon signe : il est bon de savoir que les gouvernants ne sont pas plus raisonnables que le commun des mortels du seul fait d'être des gouvernants, qu'ils soient des monarques ou des élus. Que les personnes pensent à leurs intérêts propres, ce n'est pas un vice de la nature humaine, mais plutôt un signe de santé, qui est à l'origine non seulement des passions humaines mais aussi de la raison et des affects actifs.

Aussi le rôle de l'État sera-t-il de les convaincre du bien-fondé de ses actions, par la raison et non pas par d'autres passions. Et cela, à son propre avantage : l'État quitte, pour ainsi dire, un niveau de dialogue qui se situait dans l'univers des passions, où le plus souvent se crée un accord tacite selon lequel on trouve normal que tous, peuple et gouvernants, essaient de tirer parti le plus passionnellement possible du pouvoir qui leur est accordé, même si cela se fait au nom de la raison. En abandonnant ce niveau d'accord tacite moral (voire même immoral), l'État gagne de la crédibilité et peut donner l'exemple de désirs naissant de causes adéquates, en montrant que mieux vaut des actions éthiques bien assises dans la réalité, que des idéaux moraux qui parfois ne font que cacher des propos mesquins, tout compte fait tolérés. L'État se fait par là même respecter, gagnant donc la sympathie, la confiance et la fidélité de son peuple, même si les personnes y voient une façon de faire raisonnée et éthique assez inaccessible pour eux. Par l'exemple de gouvernants éthiques, bien qu'ils puissent paraître un modèle éloigné du peuple, les personnes deviendront au moins un peu plus conscientes de leurs passions et s'écarteront de l'idolâtrie.

En bref, il n'est pas raisonnable qu'au nom de la raison, un État profère des menaces, agisse par la force ou la violence contre les peuples, fomente les croyances religieuses. S'il doit se garder d'être renversé par les passions de son peuple, ou par des passions de ses concurrents qui peuvent inciter son peuple à «lutter pour sa servitude comme si c'était pour sa liberté », manipulé par des intérêts contraires aux siens propres, il doit le faire non pas par le moyen de passions encore plus puissantes, et pas plus par un raisonnement prétendument «pur»-puisqu'un tel raisonnement ne serait pas assez raisonnable : ce serait un idéal de raison, celui d'une raison pure, non affective, non liée au corps, qui ne prendrait pas en compte « les raisons du corps » et ses affects. Une raison qui se veut «pure » pourrait arriver, par exemple, à la conclusion que la sécurité d'un État doit se faire même au détriment de la puissance ou de l'expression de ses membres en général, ou même par des menaces, des tortures, des persécutions. Une telle raison, si l'on peut toujours l'appeler ainsi, se concevrait comme séparée du monde sensible, de la réalité qui est en fait affective et vécue par des êtres singuliers. En revanche, prendre en compte les affects, donc les affections, les désirs et les appétits, et le fait qu'ils existent, fait alors partie intégrante d'une raison affective adéquate à la substance, à la fois pensée et étendue. Convaincre le peuple pris dans ses passions exige donc non pas des passions encore plus fortes - car ce serait toujours une arme à double tranchant -, mais un souci prononcé de montrer que les raisons de l'État sont désirables et profitables à tous. Même si l'on considère que la raison s'exprime le plus 
proprement par la voie de la logique et du formalisme, une exigence de communication s'ajoute à l'exigence rationnelle si l'on veut transmettre la raison à autrui et surtout à un grand nombre. Cette communication ne doit pas forcément exploiter les passions, même si elle doit prendre en compte les affects et les désirs et se servir de l'imaginaire et de l'expérience. Tout comme dans les appendices de l'Éthique, ou même les scolies ou certaines démonstrations, Spinoza utilise des exemples tirés de l'expérience et des analogies pour mieux se faire comprendre, tout en gardant le fil de la raison et par conséquent en évitant les idées inadéquates.

En un mot, la raison doit être attirante, convaincante, pour être effectivement transmise et acquise ${ }^{28}$. Elle doit se servir des images et de l'imagination, mais non pas des idées fausses et trompeuses, y compris celle d'un libre-arbitre ou d'une raison formellement universelle, abstraite et détachée du monde, comme si l'État était l'esprit éclairé de la collectivité et la multitude, son corps. La question n'est donc pas d'être pour ou contre la diffusion attrayante d'une idée, d'une prise de décision ou d'un plan d'action, mais il s'agit de comprendre que par la même voie de la communication attrayante d'une idée, un État peut, il est vrai, tromper les personnes, mais aussi les aider à devenir critiques et autonomes et donc à être à même de chercher ce qui leur est utile par des idées de plus en plus adéquates - même si cela ne mènera jamais à une harmonie absolue entre les individus, vu que chacun gardera toujours sa singularité, son ingenium, ses désirs, et d'une façon ou d'une autre toujours des passions et des appétits. Ce sera donc l'usage des moyens de persuasion et des idées qui y sont véhiculées qui pourra être ou bien moral et passionnel, voire hypocrite, ou bien éthique, à la fois rationnel et affectif, conduisant vers les idées adéquates, l'autonomie singulière et les affects actifs. La communication de la raison de façon attirante n'empêche pas les idées adéquates, ne rend pas la raison moins raisonnable, mais au contraire la rend plus efficace. Cela devient évident lorsqu'on pense que le langage est un produit du premier genre de connaissance, et qu'il est absolument nécessaire pour véhiculer et pour permettre les deuxième et troisième genres de connaissance - il devra donc être d'autant plus compréhensible, et par là même persuasif. La force du langage peut persuader d'idées fausses mais aussi d'idées vraies ; il ne faut pas penser que le fait qu'il soit persuasif rende nécessairement fausses les idées qu'il exprime. Ce serait un contresens, puisqu'il n'est pas de communication d'idées sans persuasion. Autrement dit, l'État ne doit pas forcément s'abaisser du deuxième genre de connaissance au premier, celui des passions, pour se faire comprendre du plus grand nombre, mais au contraire, s'élever jusqu'au deuxième puis au troisième en se servant du premier. Pouvoir communiquer de façon rationnelle, c'est atteindre un

28. La raison a besoin de l'imagination jusque dans la partie V de l'Éthique, lorsque Spinoza développe l'idée du troisième genre de connaissance, qui rend sensibles les raisons communes : «Plus cette connaissance que les choses sont nécessaires s'appliquent aux choses singulières, que nous imaginons de manière plus distincte et vive, plus grand est cette puissance de l'Esprit sur ses affects » (Ethique, V, prop. 6, scol. ; nous soulignons). 
niveau plus élevé de connaissance que celui d'une raison purement formelle ou universelle, marquant le passage du deuxième au troisième genre de connaissance. Se servir de l'imagination pour mieux communiquer va dans le sens opposé de se servir des passions pour s'imposer. Alors que des moyens passionnels empêchent des affects actifs et la compréhension rationnelle ou de troisième genre de la réalité, des moyens imaginatifs peuvent les favoriser.

$\mathrm{Si}$ «c'est à l'État de rendre les hommes vertueux et non d'attendre qu'ils le soient ${ }^{29}$, comme le souligne Moreau, il faut comprendre que l'État ne peut pas, bien entendu, «rendre les hommes vertueux » par la force du libre-arbitre ou d'un acte volontariste. Il peut, par contre, tenter de favoriser la vertu par des actions et des prises de décision planifiées qui puissent être efficaces dans la pratique. Or, rappelle Jaquet, le «salut » ne dépend pas de la politique, mais «du conatus propre à l'individu et de l'existence inévitable des causes extérieures ${ }^{30}$. Dans ce carrefour de rencontres et d'influences, l'État peut toutefois jouer un rôle important pour mettre en place des conditions favorables à l'autonomie individuelle. S'il est vrai que « le mythe de l'état de nature et du contrat ne sert qu'à placer dans l'individu et sa libre volonté ce que de toute façon il faut retrouver, puisqu'il y est déjà : l'État et la contrainte qu'il exerce $»^{31}$, il faut alors penser un État où cette contrainte inhibe les passions (allant contre, par exemple, les actions guidées par la ruse, la colère ou la haine), mais le moins possible les affects actifs de ses membres - l'État doit, au contraire, soutenir l'autonomie de leur pensée et de leur action. «La fin de l'État est donc en réalité la liberté ${ }^{32}$, conclut Spinoza. C'est en ce sens que la démocratie est le genre d'État qui pourrait fonctionner effectivement de la façon la plus éthique. «La meilleure société sera donc celle qui exempte la puissance de penser du devoir d'obéir $»^{33}$, celle qui, finalement, même si la foule réagit la plupart du temps de manière passionnelle, permet aux hommes de vivre et de s'épanouir, d'être dans leur droit naturel et leur puissance propre d'agir, amenuisant les affects passifs, favorisant les affects actifs et l'éthique.

29. P.-F. Moreau, Spinoza et le spinozisme, op. cit., p. 88.

30. C. Jaquet, Spinoza ou la prudence, Paris, Quintette, 1997, p. 68.

31. P.-F. Moreau, Spinoza, op. cit., p.115.

32. Spinoza, Traité théologico-politique, chap. 20, p. 329.

33. G. Deleuze, Spinoza, philosophie pratique, Paris, Minuit, 1980, p. 10. 